\title{
Cricoid ridge ossification mimicking ingested fish bone on plain radiography: prevalence in Singapore
}

\author{
Sheldon Jin Keat $\underline{\mathrm{Ng}}^{1}$, MBBS, FRCR, Joseph King Tak $\underline{\operatorname{Lee}}^{1,2}$, MD, FACR, Yee Liang $\underline{\operatorname{Thian}}^{1}$, MBBS, FRCR
}

INTRODUCTION Ossification of the cricoid ridge mimicking impacted upper aerodigestive tract fish bone has been described in several case reports. We performed this study to determine the prevalence of such ossification in an adult population from Singapore and to identify specific imaging features that may aid in differentiating patients with cricoid ridge ossification from those with an upper aerodigestive tract impacted fish bone.

METHODS We retrospectively evaluated 442 lateral neck radiographs of patients aged 16 years and above that were taken at the adult emergency department at National University Hospital, Singapore, during the four-month period between 1 September 2014 and 31 December 2014.

RESULTS Ossification of the cricoid ridge mimicking fish bone appeared in $3.2 \%$ of our adult patients and was more common among patients aged above 40 years $(n=11 / 227 ; 4.8 \%)$. The ossified cricoid ridge was usually located at the C6 level $(n=9)$ and had a mean length of 5.5 (range 2-9) $\mathrm{mm}$.

CONCLUSION Ossified cricoid ridges may be misinterpreted on lateral neck radiography as ingested fish bones. While ossification of the cricoid ridge mimicking fish bones is rare, awareness of such ossification would reduce unnecessary further investigations of the upper aerodigestive tract in patients suspected of having an impacted foreign body.

Keywords: cricoid, fish bone, ossification, Singapore

\section{INTRODUCTION}

In Singapore, as well as many other countries in Asia, it is common to eat a whole fish with intact bones, and as a result, patients frequently present to primary care physicians and emergency departments (EDs) with suspected fish bone ingestion and impaction in the upper aerodigestive tract. Apart from thorough clinical history-taking and physical examination, lateral radiography of the neck is frequently performed as initial evaluation for this group of patients.

Vertical ossification of the cricoid ridge (i.e. posterior midline of the cricoid lamina), also known as vertical cricoid lamina, is highly variable. When it occurs, it may be misdiagnosed on lateral neck radiographs as an ingested fish bone. A literature search showed that fewer than ten isolated case reports have been published in the English-language literature and were mainly from Asian countries. ${ }^{(1-7)}$ However, to the best of our knowledge, the frequency of such an occurrence has not been studied in any patient population. We performed this study to: (a) determine the prevalence of such ossification of the cricoid ridge in the local adult population; and (b) identify specific imaging features that can be used as an aid for differentiating patients with cricoid ridge ossification from those who have an upper aerodigestive tract impacted fish bone.

\section{METHODS}

A total of 466 consecutive lateral neck radiographs of patients aged 16 years and above that were performed in the adult ED at National University Hospital, Singapore, during the four-month period from
1 September 2014 to 31 December 2014 were retrospectively evaluated. Institutional review board approval and waiver of informed consent were obtained for this retrospective study.

Lateral neck radiography was performed using the AGFA DX-G (Siemens AG, Erlangen, Bavaria, Germany) computed radiography system. The standard projection was a single lateral view, with the patient positioned erect and neck extended in full inspiration, exposure of $60 \mathrm{kVp}$ and $6 \mathrm{mAs}$, and the beam centred at the thyroid cartilage (level of C4 vertebra). External artefacts, such as jewellery and clothing, were removed before the examination.

The radiographs in this study were analysed concurrently by a senior consultant radiologist with 37 years of experience and a senior resident in the fifth year of radiology training, on a GE PACS (GE Healthcare, Little Chalfont, UK) workstation. A positive finding was defined as dense linear ossification of the cricoid ridge that appeared separate from the rest of the partially or completely ossified cricoid cartilage. The positive finding had to be agreed upon by both readers, and the clinical notes of patients were then reviewed to confirm that it was not a foreign body. During this study, no disagreements occurred between the two readers regarding any positive radiographic findings. A foreign body was excluded based on resolution of symptoms, and negative endoscopy of the oropharynx and hypopharynx. We also analysed the location, length, width and density (by measuring optical density with an electronic cursor and comparing with the cervical vertebrae cortex) of the cricoid ridge ossification, as well as the ossification pattern of the rest of the laryngeal cartilages in patients with positive findings. The radiographic findings of cricoid ridge

${ }^{1}$ Department of Diagnostic Imaging, National University Hospital, Singapore, ${ }^{2}$ Department of Radiology, School of Medicine, University of North Carolina at Chapel Hill, North Carolina, USA

Correspondence: Dr Ng Sheldon Jin Keat, Associate Consultant, Department of Diagnostic Imaging, National University Hospital, 5 Lower Kent Ridge Road, Singapore 119074. Sheldon_jk_ng@nuhs.edu.sg 
Table I. Patients with cricoid ridge ossification.

\begin{tabular}{|c|c|c|c|c|}
\hline \multirow[t]{3}{*}{ Age range (yr) } & \multicolumn{4}{|c|}{ No. (\%) } \\
\hline & \multicolumn{2}{|c|}{ Total $(n=442)$} & \multicolumn{2}{|c|}{ Cricoid ridge ossification $(n=14)$} \\
\hline & Male $(n=242)$ & Female $(n=200)$ & Male $(n=7)$ & Female $(n=7)$ \\
\hline $16-20$ & $17(7.0)$ & $17(8.5)$ & - & - \\
\hline $21-40$ & $109(45.0)$ & $72(36.0)$ & $2(28.6)$ & $1(14.3)$ \\
\hline $41-60$ & $65(26.9)$ & $64(32.0)$ & $4(57.1)$ & $4(57.1)$ \\
\hline $61-80$ & $44(18.2)$ & $42(21.0)$ & $1(14.3)$ & $2(28.6)$ \\
\hline$>81$ & $7(2.9)$ & $5(2.5)$ & - & - \\
\hline
\end{tabular}
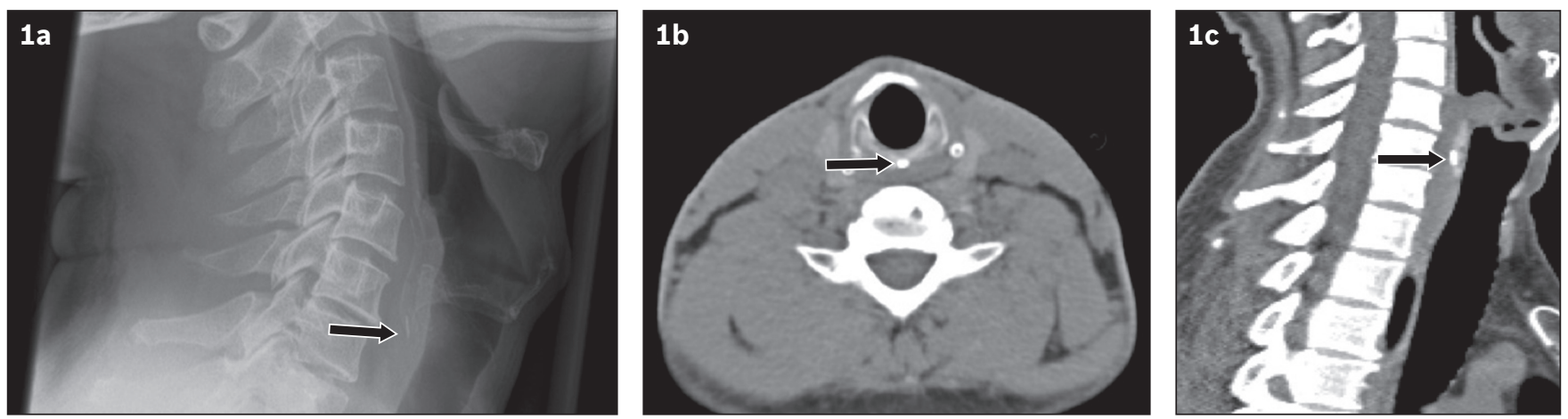

Fig. 1 A patient was suspected of having an impacted fish bone that was found to be an ossified cricoid ridge on further examination. (a) Lateral neck radiograph showed linear ossification at the posterior cricoid (arrow), which was initially thought to be an impacted fish bone. Unenhanced CT images in the (b) axial and (c) sagittal planes showed an ossified cricoid ridge (arrows).
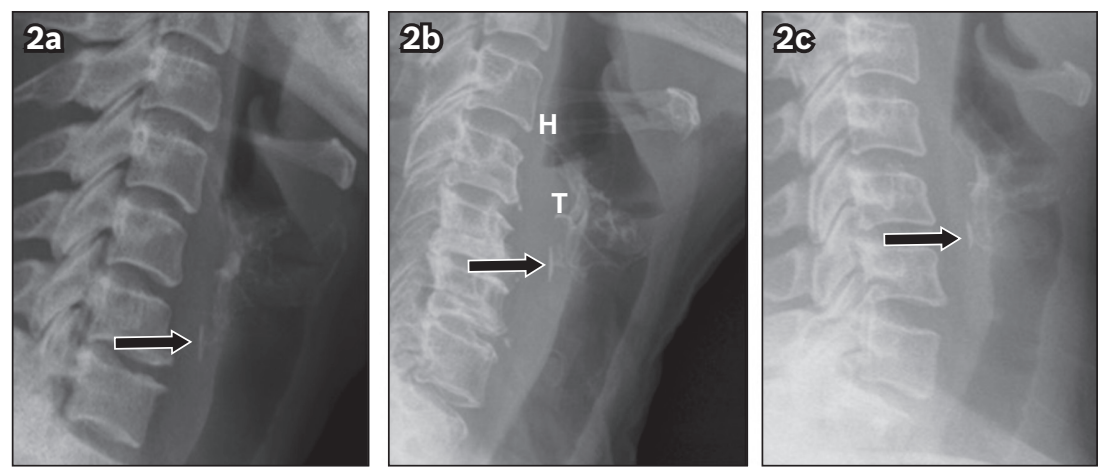

Fig. 2 Lateral neck radiographs of three patients show ossification of the cricoid ridge (arrow) that appeared separate from the rest of the cricoid. $\mathrm{H}$ : hyoid bone; T: partially ossified thyroid cartilage

ossification were then compared to that of patients with proven impacted fish bone in a similar location in our study group.

All statistical analyses were performed using Stata 12 (StataCorp LP, College Station, TX, USA). Descriptive statistics were obtained to assess the frequency and characteristics of cricoid ridge ossification. Chi-square test was used to assess the association between age group and occurrence of cricoid ridge ossification. A p-value $\leq 0.05$ was considered to be statistically significant.

\section{RESULTS}

Among the 466 radiographs studied, 24 radiographs were excluded, as they were repeat radiographs of the same patients (22 patients had two radiographs, and one patient had three radiographs). The final number of patients included in our study was 442 .

Their age range was 16-92 years (242 male, 200 female) (Table I). The most common indication for performing lateral neck radiography in the $\mathrm{ED}$ was for further evaluation of suspected foreign body impaction in the upper aerodigestive tract. Other less common indications included suspected epiglottitis and retropharyngeal abscess.

Of the 442 patients included in the study, 14 had ossification of the cricoid ridge that appeared separate from the rest of the partially or completely ossified cricoid cartilage (Figs. $1 \& 2$ ). The prevalence of this pattern of ossification was 3.2\% (14/442 patients) in our sample study population. There was an equal number of male $(n=7)$ and female $(n=7)$ patients with ossified cricoid ridges. The median age of patients with cricoid ridge ossification was 50 (range 34-71) years.

Due to the small number of patients in each of the three age groups (21-40 years; 41-60 years; and 61-80 years) of patients with cricoid ridge ossification, statistical analysis could only be performed by dividing the study population into patients aged $\leq 40$ years and $>40$ years. We found that there was a statistically 

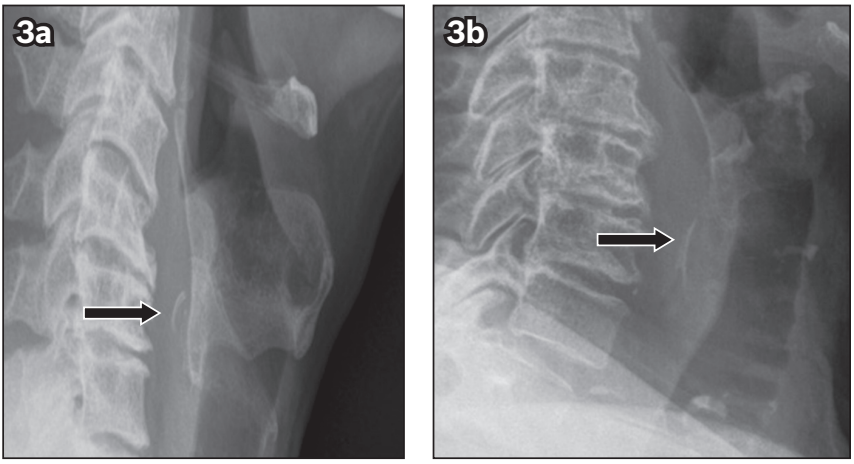

Fig. 3 Lateral neck radiographs of two patients show unusually ossified cricoid ridges that were endoscopically proven to be impacted fish bone (arrows) in the upper oesophagus. Note the (a) curved appearance and (b) inverted ' $V$ ' shape of the fish bones, in contrast to the linear appearance of normal cricoid ridge ossification.

significant higher prevalence of cricoid ridge ossification in the group aged $>40$ years $(11 / 227$ patients, $4.8 \%)$ when compared to those aged $\leq 40$ years $(0 / 215$ patients, $0 \% ; p=0.038)$.

The most common location of cricoid ridge ossification was at the C6 level $(n=9)$, followed by C5/C6 $(n=3)$ and C5 $(n=2)$. The mean length of ossification was 5.5 (range 2-9) $\mathrm{mm}$. The ossification was linear in all patients, with a width of $\leq 1 \mathrm{~mm}$. Among patients with cricoid ridge ossification, the rest of the cricoid and thyroid cartilages were partially ossified in 12 patients and completely ossified in two patients. The ossification density was $\leq 10 \%$ difference compared to the cervical vertebral body cortex in 13 patients, and 20\% less dense than the cortex in the remaining patient.

In our study population, three out of 442 patients were confirmed to have impacted fish bone in the upper oesophagus (Fig. 3). In all three patients, the fish bone was vertical in orientation, extending beyond the margins of the cricoid cartilage, with an irregular shape and varying in thickness (range 1.5-2.0 mm).

\section{DISCUSSION}

Eating fish with intact bones is common in Singapore and several other Asian countries, and therefore it is fairly common for patients to present to primary care physicians and the ED for suspected fish bone ingestion and impaction in the upper aerodigestive tract. Initial evaluation for these patients, such as thorough clinical history-taking, physical examination and lateral radiography of the neck, are frequently performed to determine the presence or absence of impacted fish bone as well as guide endoscopic examination of the oropharynx and hypopharynx in the ED for patients with persistent symptoms.

Lateral view radiography of the neck has an important role to play in the detection of upper aerodigestive tract impacted fish bone, as up to $88 \%$ of fish bones are reported to be radiopaque. ${ }^{(8)}$ Early diagnosis and management of the impacted foreign body is important to prevent complications, such as perforation with associated abscess, as well as vascular complications such as carotid puncture and aorto-oesophageal fistula. ${ }^{(9)}$

Since the initial study by Chievitz ${ }^{(10)}$ in 1882 , the calcification and ossification of laryngeal cartilages have been extensively investigated. As part of the normal ageing process, the thyroid, cricoid and arytenoid cartilages, which are made of hyaline cartilage, undergo calcification and subsequent ossification. Ossification of the laryngeal cartilages begins at the age of 20 years and 22 years in men and women, respectively. Ossification of the cricoid cartilage begins at the superior border of the lamina ${ }^{(11,12)}$ and progressive ossification of the rest of the cricoid cartilage occurs in the cranial-to-caudal and posteriorto-anterior directions. The two areas of ossification in the cricoid that are commonly mistaken for a foreign body are the superior tip of the cricoid lamina and cricoid ridge, which are known to ossify separately from the rest of the cricoid. ${ }^{(11,13)}$

Ossification of the cricoid ridge mimicking an ingested fish bone on lateral neck radiographs has been described in less than ten case reports in the English-language literature, to our knowledge, and these studies are mainly from Asian countries. ${ }^{(1-7)}$ Although this imaging pitfall may be better known to practising clinicians and radiologists from this region, the exact frequency of such a pattern of ossification has never been established. In our study of patients suspected to have ingested fish bone, the estimated prevalence in the Singapore adult population was estimated to be about $3.2 \%$ and was more common in patients aged above 40 years ( $4.8 \%)$. We believe that this number should apply to the general Singapore population as well, including patients who do not eat a whole fish with intact bones, since they are likely to share a similar pattern of cricoid ossification.

Physiological ossification of the cricoid ridge is usually thin, linear, and does not extend beyond both the superior and inferior margins of the cricoid cartilage. ${ }^{(4)}$ In addition, in our study, the most common location of cricoid ridge ossification was at the C6 level, with a mean length of $5.5 \mathrm{~mm}$, and usually equal in density to the cervical vertebral cortex. In contrast, impacted fish bone may extend beyond the margins of the cricoid cartilage, and have irregular shape, thickness and density depending on the species of the fish eaten. ${ }^{(4,14)}$ In addition, impacted fish bone may cause secondary signs, such as widening of prevertebral soft tissue.

With the increase in global travelling and the increased number of immigrants from Asian countries living in Europe and the United States, awareness of such physiological ossification mimicking an ingested fish bone is important, not only for radiologists in Asia but also for those practising in other continents, lest it be misinterpreted as ingested fish bone. While recognising the relative infrequency of this ossification, we nevertheless believe that such awareness may reduce unnecessary investigations and invasive procedures or surgery in up to $3.2 \%$ of our population. We recommend that computed tomography or complete endoscopy (oesophagoscopy) be reserved for difficult or equivocal cases where clinical suspicion (e.g. symptoms) of impacted fish bone remains strong in spite of initial negative lateral neck radiography and endoscopy of the oropharynx and hypopharynx, or for patients who have persistent symptoms despite the typical appearance of cricoid ridge ossification.

Our study had several limitations. First, the number of patients studied was relatively small for determining normal variants of cricoid ridge ossification. Second, because of the patient referral 
pattern to our hospital, we only examined patients who lived in the west of Singapore. It is possible that the frequency of cricoid ridge ossification may be different in patients from other areas of the country, although no obvious difference in patient demography exists across Singapore. Third, only three patients had impacted fish bone in our study population. A fish bone impacted at the C6 level may potentially simulate cricoid ridge ossification, even if we did not encounter such a case in our study. As previous studies have shown that different types of fish bones may have different radiodensities and appearance, ${ }^{(8,14)}$ there is a possibility that the unique distinguishing features described herein may not apply to fish from other regions. However, determining distinguishing features between cricoid ridge ossification and impacted fish bones was only secondary to our study.

In spite of the above limitations, our study was able to establish the relative frequency of cricoid ridge ossification in the Singapore adult population and highlighted some features that may be helpful in distinguishing it from impacted fish bone.

\section{REFERENCES}

1. Wakisaka N, Miwa T, Yoshizaki T, Furukawa M. Cricoid ossification mimicking an impacted foreign body. J Laryngol Otol 2006; 120:E24.
2. Laguna D, González FM. Calcification of the posterior cricoid lamina simulating a foreign body in the aerodigestive tract (2005:11b). Eur Radiol 2006; 16:515-7.

3. Arasaratnam S, Abdullah BJ, Fernandez V. Calcified vertical plate of the cricoid-a rare pitfall in the diagnosis of an oesophageal foreign body. Med J Malaysia 1998; 53:290-2.

4. Lim CT, Tan KP, Stanley RE. Cricoid calcification mimicking an impacted foreign body. Ann Otol Rhinol Laryngol 1993; 102:735-7.

5. McDonald M, Pogrel MA. Calcification of the cricoid cartilage mistaken for a foreign body: report of a case. J Oral Maxillofac Surg 1992; 50:62-3.

6. Divya GM, Muhammad H, Ramachandran KC, Shiji K. Vertical cricoid lamina ossification: a rare pitfall in the diagnosis of extraluminal foreign body. Int J Head Neck Surg 2014; 5:91-3.

7. Raza S, Asrar L, Oyetunji N, Al Belaihi M, Kakaria A. Calcified posterior part of cricoid cartillage presenting as foreign body complicated with retropharyngeal cellulitis. J Pak Med Assoc 2004; 54:224-6.

8. Abdullah BJ, Ng KH, Kaur H. Radio-opacity of Malaysian fish bones: an in vitro study. Asian J Radiol 1997; 3:327-38.

9. Palme CE, Lowinger D, Peterson AJ. Fish bones at the cricopharyngeus: a comparison of plain-film radiography and computed tomography. Laryngoscope 1999; 109:1955-8.

10. Chievitz JH. [Untersuchungen über die Verknöcherung der menschlichen Kehlknorpel]. Arch Anat Physiol Anat Abt 1882; 49:303-49. German.

11. Hatley W, Samuel E, Evison G. The pattern of ossification in the laryngeal cartilages: a radiological study. Br J Radiol 1965; 38:585-91.

12. Keen JA, Wainwright J. Ossification of the thyroid, cricoid and arytenoids cartilages. S Afr J Lab Clin Med 1958; 4:83-108.

13. Richardson GS. Unusual calcification of cricoid cartilage masquerading as foreign body in esophagus. AMA Arch Otolaryngol 1955; 62:316-8.

14. Ell SR, Sprigg A. The radio-opacity of fish bones--species variation. Clin Radiol $1991 ; 44: 104-7$. 